\title{
Coupled fluid-flow and magnetic-field simulation of the Riga dynamo experiment
}

\author{
S. Kenjereš, K. Hanjalić, and S. Renaudier \\ Department of Multi Scale Physics and J.M. Burgerscentre for Fluid Dynamics, \\ Delft University of Technology, Lorentzweg 1, 2628 CJ Delft, The Netherlands \\ F. Stefani and G. Gerbeth \\ Forschungszentrum Rossendorf, P.O. Box 510119, 01314 Dresden, Germany
}

A. Gailitis

Institute of Physics, University of Latvia, LV-2169 Salaspils 1, Riga, Latvia

(Received 16 June 2006; accepted 13 November 2006; published online 26 December 2006)

\begin{abstract}
Magnetic fields of planets, stars, and galaxies result from self-excitation in moving electroconducting fluids, also known as the dynamo effect. This phenomenon was recently experimentally confirmed in the Riga dynamo experiment [A. Gailitis et al., Phys. Rev. Lett. 84, 4365 (2000); A. Gailitis et al., Physics of Plasmas 11, 2838 (2004)], consisting of a helical motion of sodium in a long pipe followed by a straight backflow in a surrounding annular passage, which provided adequate conditions for magnetic-field self-excitation. In this paper, a first attempt to simulate computationally the Riga experiment is reported. The velocity and turbulence fields are modeled by a finite-volume Navier-Stokes solver using a Reynolds-averaged-Navier-Stokes turbulence model. The magnetic field is computed by an Adams-Bashforth finite-difference solver. The coupling of the two computational codes, although performed sequentially, provides an improved understanding of the interaction between the fluid velocity and magnetic fields in the saturation regime of the Riga dynamo experiment under realistic working conditions.
\end{abstract}

(C) 2006 American Institute of Physics. [DOI: 10.1063/1.2404930]

\section{INTRODUCTION}

Magnetic-field generation in cosmic bodies such as planets, stars, and galaxies, originates from the homogeneous dynamo effect in moving conductors. ${ }^{1-3}$ As a typical bifurcation phenomenon, this effect occurs only when a critical dimensionless number is reached. The relevant number for dynamos is the magnetic Reynolds number $\operatorname{Re}_{m}:=\mu_{0} \sigma l v$, where $\mu_{0}$ denotes the magnetic permeability of the fluid, $\sigma$ its electrical conductivity, $l$ a typical length scale, and $v$ a typical velocity scale of the flow. The critical value depends on the flow pattern, which is controlled by the hydrodynamic Reynolds number Re $:=v \cdot l / \nu$ (where $\nu$ is the kinematic viscosity of the fluid).

The first experimental demonstration of the homogeneous dynamo effect traces back to the 1960s, when Lowes and Wilkinson carried out experiments with two cylinders rotating around inclined axes within a block of iron (see Ref. 4 for the history of these experiments). Besides the fact that these experiments were strongly dominated by the high permeability of the used materials, their mechanical stiffness made them unsuitable for the investigation of nontrivial saturation effects.

Dynamos of genuine hydromagnetic character were first realized in laboratory in 1999, when the liquid sodium facilities in Riga and Karlsruhe became operative. ${ }^{5,6}$ Since then, a number of experiments have been carried out at both places providing a wealth of data on the dynamo behavior in the kinematic and the saturation regime. ${ }^{7-18}$ Further dynamorelated experiments are being prepared or carried out at vari- ous places in the world. ${ }^{19-23}$ Although, up to present, they have not yet reached the self-excitation condition, they have brought about many interesting results, in particular concerning the role of turbulence in flows with large $\mathrm{Re}_{m}$.

The present paper is exclusively devoted to the Riga dynamo experiment. As a follow up of a preceding paper, ${ }^{14}$ we aim at an improved numerical simulation of the interaction of velocity and magnetic field. In contrast to Ref. 14, where the velocity field was assumed to be known by preceding measurements in a water test facility, our first goal is now to simulate the fluid flow numerically. With the given Reynolds number of $\operatorname{Re} \approx 3.5 \times 10^{6}$ this cannot be done (at present) by a direct numerical simulation (DNS). The central part of the paper is, therefore, devoted to a Reynoldsaveraged-Navier-Stokes (RANS) model for the flow in the realistic geometry of the Riga dynamo.

At this point, we would like to underline the increasing importance of turbulence modeling for the simulation of hydromagnetic dynamos in general, and of the geodynamo in particular. It is certainly true that impressive numerical results, including the occurrence of polarity reversals, have been achieved by a number of fully coupled threedimensional (3D) geodynamo models during the past decade. ${ }^{24}$ However, most of these models are still working in parameter regions far away from those of the Earth and/or with rather oversimplified models of turbulence, such as artificial hyperviscosities. Only recently has more effort been spent on applying up-to-date methods of subgrid scale modeling to the geodynamo, including large-eddy-simulation (LES) models ${ }^{25,26}$ and RANS models. ${ }^{27}$ A central problem 
for turbulence modeling of magnetohydrodynamics (MHD) flows at large $\operatorname{Re}_{m}$ (with and without strong rotation) is that there are not many experiments on which they could be checked. This present paper is a first approach to do exactly this by validating an existing MHD RANS model on the Riga dynamo experiment.

A significant part of the paper is devoted to the RANS simulation of the pure hydrodynamic loop of the Riga dynamo. At the relevant Reynolds number this is already a formidable problem which must be successfully solved before the very MHD regime can be entered. We will see that the used RANS model gives a good description of the velocity profiles that were measured at a 1:2 water test loop of the Riga dynamo facility.

As for the coupled MHD problem, our work represents a compromise in the form of the coupling of a RANS model with an older Adams-Bashforth finite-difference solver that was described in Refs. 14 and 28. The reason is twofold: First, as the ratio $\operatorname{Re}_{m} / \operatorname{Re} \approx 10^{-5}$, the effective length scales of the magnetic field are much larger than for the velocity field. This means that only a comparably coarse grid is needed to get a physically correct picture of the magnetic field (a similar argument can be found in Ref. 29).

Second, the correct implementation of the nonlocal boundary conditions for the magnetic field is a notorious problem in nonspherical geometries. In the employed finitedifference solver this problem was overcome by solving the Laplace equation in the exterior and using matching conditions at the interface to the dynamo domain. ${ }^{14,28} \mathrm{~A}$ similar approach, although based on the finite element method, was presented by Guermond et al. ${ }^{30}$ Other methods of handling this boundary condition problem are the integral equation approach $\mathrm{c}^{31-33}$ and a hybrid boundary element/finite volume method. ${ }^{34}$ For the Riga experiment, we also checked the use of simplified boundary conditions (so-called vertical field conditions $^{35}$ ) which led, however, to a significant $20 \%$ error in the determination of the critical $\mathrm{Re}_{m}$. Hence, such simplified boundary conditions should not be used in the simulation of a real experiment.

Consequently, in order to concentrate on the RANS modeling, and not to be distracted by the intricacies of the boundary condition problem for the magnetic field, we decided to use a coupling of two codes for the determination of the fluid flow and the magnetic field. Of course, a simultaneous simulation of both quantities in one common code would certainly be desirable, since an obvious disadvantage of such a coupling is the necessity to project the result of either code to the different geometry of the other, which needs some additional effort. Such a simultaneous simulation is left for future work.

One of the most interesting questions of dynamo research is the understanding of the saturation mechanism. This relies basically on Lenz's rule stating that the generated magnetic field acts (via the Lorentz force) against the source of its own generation. In a first attempt to quantify the main saturation effect, we had developed a very simple onedimensional back-reaction model that relies on the accumulative braking of the azimuthal velocity component along the vertical axis of the dynamo. ${ }^{11,14}$ This simple model, which

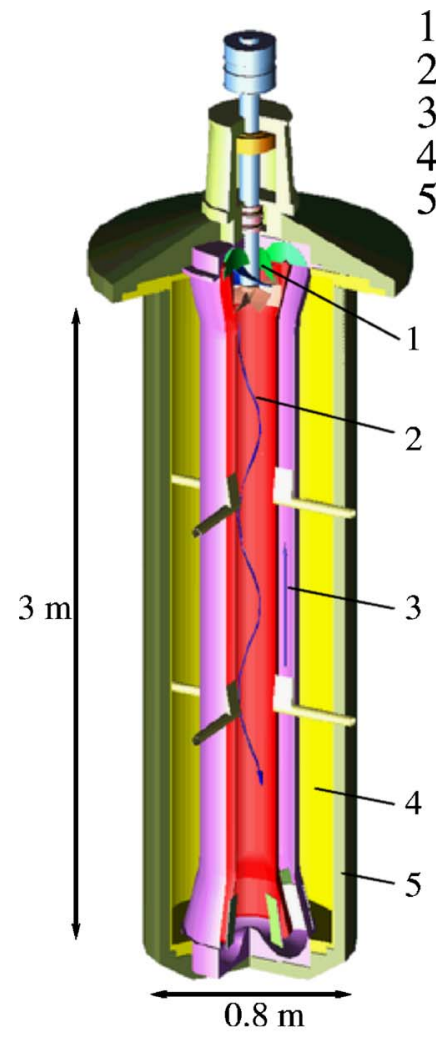

(a)
1 - Propeller

2 - Helical sodium flow

3 - Back flow

4 - Sodium at rest

5 - Thermal insulation

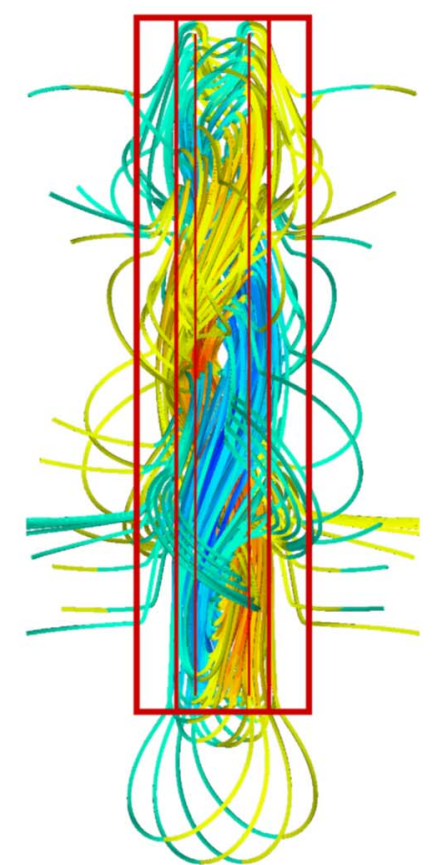

(b)
FIG. 1. (Color online) The Riga dynamo experiment. (a) Central module of the facility. (b) Snapshot of the "double-helix" magnetic-field structure resulting from the undisturbed velocity field. The color scale of the magneticfield lines indicates the axial component of the field.

gave already a reasonable self-consistent picture of magnetic-field strength, Joule excess power, growth rate, and frequency in the saturation regime, will be refined in the present paper.

\section{EXPERIMENTAL BACKGROUND}

The "heart" of the Riga dynamo experiment consists of three concentric cylinders with different flow structures (Fig. 1). In the central cylinder the liquid sodium is pumped by a propeller on a helical path with a carefully adjusted relation of the axial and azimuthal velocity components. ${ }^{28,36,37}$ The optimization of the radial dependence of both components aimed at maximization of the flow helicity. ${ }^{28}$ Evidently, in the real experiment the demanded Bessel function profiles can never be realized exactly. Nevertheless, a maximum of the axial velocity at the center, and a maximum of the azimuthal velocity close to the inner wall, are well realizable by a suitable configuration of prepropeller and postpropeller vanes.

The propeller, in turn, is driven by two electric motors with a power that can reach up to $100 \mathrm{~kW}$ each. After the swirl is removed by guide vanes placed in the lower bend of the rig, the flow becomes basically rotationless in the backflow annular passage. However, the back reaction of the self- 
excited magnetic field is supposed to induce some rotation within this tube. The same holds for the outermost cylinder where the sodium is stagnant at the beginning of the experiment, but where the Lorentz forces are also expected to drive some flow when the magnetic field has become strong enough. The total volume of the used sodium is $2 \mathrm{~m}^{3}$. The velocity reaches values up to $20 \mathrm{~m} / \mathrm{s}$. Needless to say, special care is necessary to run a sodium facility of that size in a safe manner. During the experiment, the magnetic field is measured by flux-gate sensors, Hall sensors, and induction coils.

\section{NUMERICAL CODES}

\section{A. Summary of previous numerical efforts}

The idea of the Riga dynamo experiment traces back to 1973, when Ponomarenko ${ }^{38}$ had proved dynamo action for a conducting rod moving on a helical path through a conducting medium of infinite extension. The kinematic regime of the Riga dynamo experiment has been extensively studied by various one-dimensional (in $r$ ) (Refs. 39-41) and twodimensional (2D) (in $r-z$ ) $\operatorname{codes}^{14,28}$ for the solution of the induction equation,

$$
\frac{\partial \mathbf{B}}{\partial t}=\nabla \times(\mathbf{v} \times \mathbf{B})+\frac{1}{\mu_{0} \sigma} \Delta \mathbf{B} .
$$

The two-dimensional code, which is essentially a finitedifference solver using Adams-Bashforth method for the time integration, will be used in the following to obtain the magnetic field and Lorentz force structure which enter the RANS model for the velocity.

In a first attempt to elucidate the basic physical mechanism of the saturation, we restricted ourselves to the braking and accelerating effect of the Lorentz forces on the azimuthal velocity field. ${ }^{11,14}$ In doing so, the pressure increase due to the axial Lorentz force component is taken into account by calibrating the magnetic-field amplitude in such a way as to fit the resulting Joule losses to the measured excess power of the motors. Adopting the weak-field dynamo concept, using the inviscid approximation, and considering only the $m=0$ mode of the Lorentz force, we ended up with the ordinary differential equation for the perturbation $\delta v_{\phi}$,

$$
\bar{v}_{z} \frac{\partial}{\partial z} \delta v_{\phi}=\frac{1}{\mu_{0} \rho}[(\nabla \times \mathbf{B}) \times \mathbf{B}]_{\phi},
$$

which describes the downward braking of the azimuthal velocity in the central channel and the upward acceleration in the backflow channel. This equation can easily be solved when the azimuthal Lorentz force component is known.

This simple saturation model serves us in the following as the basis with which we will compare the results of the RANS model.

\section{B. RANS model}

\section{Mathematical rationale and turbulence models}

The fluid flow in the Riga dynamo experiment exhibits some features that are very challenging for numerical simulations. Because of the very high $\operatorname{Re}$ number $(\operatorname{Re} \approx 3.5$

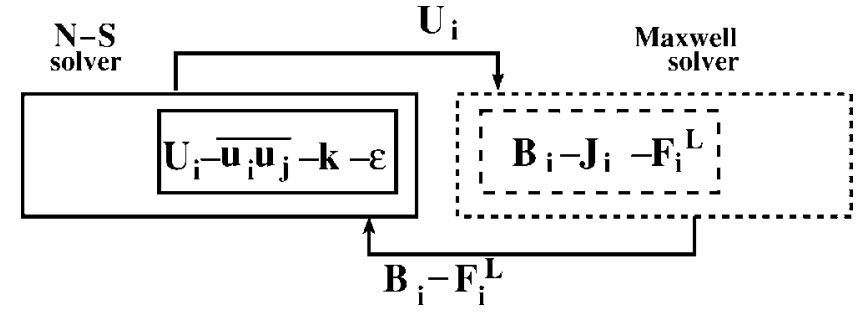

FIG. 2. Schematic overview of the fluid-flow/turbulence and electromagnetic-field interactions: coupling between Navier-Stokes and Maxwell (magnetic-field) solver.

$\left.\times 10^{6}\right)$ needed for ensuring the critical $\operatorname{Re}_{m}\left(15 \leq \operatorname{Re}_{m} \leq 20\right)$, the application of the fully resolving direct numerical simulation for this configuration is at present beyond the reach because of its enormous computational requirements. A possible alternative can be the LES approach, where only the large flow structures are resolved by numerical mesh and the smaller ones are represented through a subgrid-scale turbulence model. Unfortunately, the complexity of the geometry together with the high Re number will require still very demanding computational resources, which, in the light of the sequential solver coupling and the main target of predicting the experimentally recorded growth rates and frequencies of the self-excited magnetic field, will be hardly justified. Obviously, the only available option is to apply numerical simulations based on the RANS approach (Fig. 2).

However, accurate predictions of the dynamics of a strong swirl of sodium flow in the inner cylinder (another prerequisite for achieving critical conditions) are a major challenge for a RANS turbulence model, and so is the flow in the $180^{\circ}$ loop bend and the subsequent backflow in the annular passage. These flow features have been notoriously difficult to predict accurately with the standard eddyviscosity turbulence closures such as $k-\varepsilon$ or similar models. For this reason we considered in addition to the standard $k-\varepsilon$ model also a more advanced, full second-moment (differential Reynolds-stress) closure. In contrast to the eddyviscosity models, which provide only the turbulence kinetic energy and its dissipation rate or another scale-providing variable (used to determine the eddy viscosity), the secondmoment closure (SMC) provides the full turbulent stress tensor $\overline{u_{i} u_{j}}$. It is noted that capturing the turbulence stress field in all its components and their anisotropy is often a prerequisite for predicting accurately the velocity field in inhomogeneous flows. We anticipated that this could also be important in MHD flows where the Lorentz force, acting selectively on velocity and its fluctuations only in its direction, usually increases the stress anisotropy influencing the bulk velocity field. On the other hand, the second-moment closure requires an additional six partial differential equations to be solved (for each component of the Reynolds stress tensor, $\overline{u_{i} u_{j}}$ ). The parallel simulations of the water flow model for which the velocity measurements were available (Refs. 36 and 37), both with the $k-\varepsilon$ and second-moment (Reynolds stress) closure, were to provide an estimate of whether the more elaborate Reynolds-stress model should be 
used for the simulation of the real Riga experiment with a magnetic field, or the simpler and more economical $k-\varepsilon$ model could still suffice.

We present now a short outline of both models considered, which were used to close the RANS mean momentum equation. Together with the continuity equation $\partial U_{j} / \partial x_{j}=0$, the RANS momentum equation describing turbulent flow of an incompressible fluid subjected to a magnetic field can be written as

$$
\begin{aligned}
\frac{\partial U_{i}}{\partial t}+U_{j} \frac{\partial U_{i}}{\partial x_{j}}= & \frac{\partial}{\partial x_{j}}\left[\nu\left(\frac{\partial U_{i}}{\partial x_{j}}+\frac{\partial U_{j}}{\partial x_{i}}\right)-\overline{u_{i} u_{j}}\right]-\frac{1}{\rho} \frac{\partial P}{\partial x_{i}} \\
& +\underbrace{\frac{1}{\rho \mu_{0}}\left(B_{j} \frac{\partial B_{i}}{\partial x_{j}}-B_{j} \frac{\partial B_{j}}{\partial x_{i}}\right)}_{\mathbf{F}^{\mathbf{L}}=\mathbf{1} / \mu_{0}(\nabla \times \mathbf{B}) \times \mathbf{B}} .
\end{aligned}
$$

The turbulent stress tensor $\overline{u_{i} u_{j}}$ is provided by a turbulence model. The SMC requires the solution of the model equations for the turbulent stress tensor ${\overline{u_{i}}}_{j}$ and the dissipation rate $\varepsilon$ of the turbulent kinetic energy $k$ (see the Appendix). It is noted that when the second-moment turbulence closure is applied, the production terms $\left(P_{i j}\right)$ are treated exactly. The additional source/sink terms in the $\overline{u_{i} u_{j}}$ and $\varepsilon$ equations, $S_{i j}^{M}$ and $S_{\varepsilon}^{M}$, respectively, representing effects of the fluctuating Lorentz force on turbulence, must be included, according to Kenjereš and Hanjalic ${ }^{42,45}$ (see, also, Hanjalić and Kenjeres $\left.{ }^{43,44,47}\right)$. The redistributive terms $\left(\Phi_{i j}\right)$, the slow $\left(\Phi_{i j}^{S}\right)$ and rapid contributions $\left(\Phi_{i j}^{R}\right)$ are modeled using the model of Speziale et al. ${ }^{48}$ (RSM_SSG), extended to account for the magnetic effect by an additional rapid term $\left(\Phi_{i j}^{M}\right)$ proposed by Kenjereš et al. ${ }^{46}$

In this study, we adopted a new extended variant of the previously proposed MHD Reynolds stress model of Kenjereš et al., ${ }^{46}$ where the MHD terms have been added to the linear version of the pressure-strain correlation together with the wall-reflection terms according to Gibson and Launder. ${ }^{49}$ The choice of the Speziale et al. ${ }^{48}$ model is primarily motivated by its ability to reasonably reproduce the near-wall stress anisotropy without introducing any special wall-reflection term. This is compensated by the nonlinear representation of the rapid redistributive term.

For industrial MHD problems, Kenjereš and Hanjalić proposed an extension of the standard two-equation $k-\varepsilon$ model to account for the effects of magnetic field. With this model the turbulent stress is computed from the conventional eddy-viscosity stress-strain relationship where the turbulent viscosity $\nu_{t}=c_{\mu} k^{2} / \varepsilon$ is obtained from the solution of equations for the turbulence kinetic energy $k$ and its dissipation rate $\varepsilon$. We note that the $k-\varepsilon$ model is in principle recovered by contracting the $\overline{u_{i} u_{j}}$ equation and the stress tensor in the $\varepsilon$ equation of the above second-moment closure, with $k=0.5 \overline{u_{i} u_{i}}$. However, some small differences exist. So, in the $\varepsilon$ equation the turbulent diffusion is now expressed via the simple-gradient diffusion instead of the general-gradient hypothesis. The Lorentz force effects are incorporated through additional source/sink terms as proposed in Kenjereš and Hanjalic. ${ }^{42,45,47}$ The coefficients for this $k$ - $\varepsilon$ MHD model are given in Table II of the Appendix.

\section{Numerics for the RANS approach}

The transport equations discussed above can be written in a general form as

$$
\frac{\partial \Phi}{\partial t}+U_{j} \frac{\partial \Phi}{\partial x_{j}}=\frac{\partial}{\partial x_{j}}\left(\Gamma_{\Phi} \frac{\partial \Phi}{\partial x_{j}}\right)+S_{\Phi},
$$

where $\Phi$ is dependent variable $\left(\Phi=U_{i}\right.$ or $\overline{u_{i} u_{j}}$ or $\varepsilon$, etc.) and $\Gamma_{\Phi}$ is characteristic transport coefficient of the dependent variable and $S_{\Phi}$ represents all source/sink terms. In the finitevolume approach that is applied in this study, all terms are preintegrated over a computational cell (elementary control volume) as

$$
\int_{V} \frac{\partial \Phi}{\partial t} d V+\int_{S}\left(U_{j} \Phi-\Gamma_{\Phi} \frac{\partial \Phi}{\partial x_{j}}\right) n_{j} d S=\int_{V} S_{\Phi} d V,
$$

where the volume integration of convective and diffusive terms is replaced by a surface integration over the control volume faces by employing the Gauss-Ostrogradski divergence theorem, i.e., $\int_{V} d i v \Phi d V=\oint_{S} \Phi \cdot n d S$, where $n$ is the unit normal vector of the cell-face surface. The solver can handle three-dimensional structured multidomain, multiblock, nonorthogonal geometries. The local grid refinement is possible for predefined blocks (i.e., specific block refinement). The solver can be run in serial (single processor) or parallel mode utilizing the domain decomposition message passing interface (MPI) directives (in our case, domain decomposition is defined in the form of decomposition over multiblocks which can be part of different domains, i.e., parts belonging to fluid flow, wall domains, or external electromagnetic layers). The collocated variable arrangement and Cartesian vector and tensor components are applied for all variables. This collocated variable arrangement requires only a single set of geometrical information to be stored in contrast with the staggered variable arrangement. In order to prevent decoupling of the velocity and pressure fields the Rhie-Chow interpolation is used. The SIMPLE algorithm is used for linking iteratively corrected velocity and pressure fields. The diffusive term [the third term in Eq. (4)] of equations set is discretized by the second-order accurate central difference scheme (CDS). The monotonicity preserving total variation diminishing (TVD) scheme is used for the convective term [the second term in Eq. (4)] with the UMIST limiter. ${ }^{50}$ Integration of the time-dependent term [the first term in Eq. (4)] is performed by the fully implicit three consecutive time-steps method with second-order accuracy allowing significantly larger time steps to be used when compared to explicit time integration methods.

\section{Simulations of the 1:2 scale-down experimental setup with water}

In order to test the performance of the two turbulence models, we performed first the fluid-flow and turbulence simulations for the 1:2 scale-down setup with water as the working fluid for which a detailed experimental database exist. ${ }^{36,37}$ The flow is characterized with very high $\mathrm{Re}$ $\approx 1.25 \times 10^{6}$ (based on the mean axial velocity and diameter of the inner cylinder). The results of numerical simulations are compared with the laser-Doppler anemometer (LDA) 


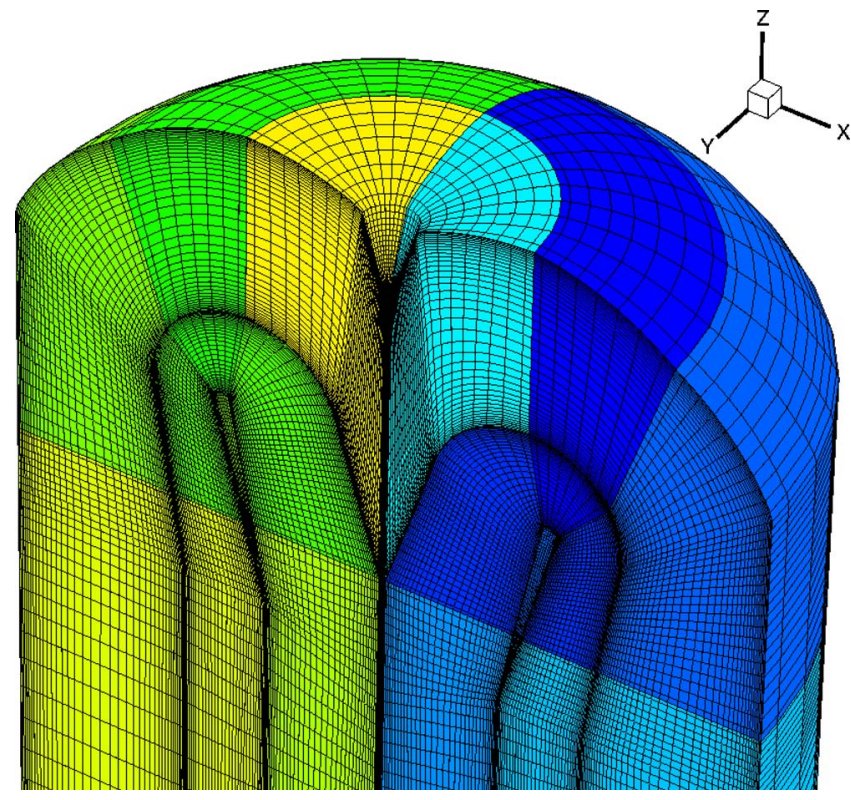

FIG. 3. (Color online) Multiblock structured numerical grid used for simulations of the Riga dynamo experimental setup: different colors are associated with different blocks. Different blocks are grouped together in order to provide the most optimal load balance when MPI directives are used. Please note that only half of the actually calculated domain is shown.

measurements performed in a vertical plane containing the axis of rotation at a few characteristic horizontal cross sections along the inner and outer flow passage. ${ }^{37}$ These measurement locations are assigned as: ME50-ME15 at $0.18 \mathrm{~m}$, ME60-ME20 at $0.465 \mathrm{~m}$, and ME75 at $0.915 \mathrm{~m}$ from the propeller location where first and second signatures are for the inner and for the outer cylinder cross sections, respectively. Both axial and azimuthal velocity components are available at these locations. The measurements are performed under the propeller working conditions at $1600 \mathrm{rpm}$.

The propeller effect (swirl generation) in numerical simulations is mimicked by imposing the axial and azimuthal velocity components (taken from measurements) at the propeller exit plane which is identical to the cylinder inlet plane. In order to avoid possible misrepresentation of the pressure field at the cylinder inlet plane, the entire reversal loop is simulated (as shown in Fig. 3). In total, the fluid part is represented by 64 multiblocks with approximately $2 \times 10^{6}$ control volumes. Numerical simulations are performed using two RANS-type turbulence models, as already discussed above. It is noted that in this case all magnetic terms in Eqs. (4)-(6) and (A1)-(A14) are switched off since the pure water flow is considered. The identical numerical resolution is employed for both eddy-viscosity $k-\varepsilon$ and full second-moment $\overline{u_{i} u_{j}}-\varepsilon$ closure (RSM_SSG). Because of the very high Re number, the standard wall functions were used for providing the wall boundary conditions for all variables.

A comparison between experimental (Refs. 36 and 37) results and numerical simulations is shown in Figs. 4-6. The axial velocity profiles in the inner cylinder show characteristic distributions with pronounced peaks along the central line $(r=0)$; Fig. 4. Numerical simulations, particularly with the full-second moment closure (RSM_SSG), show good
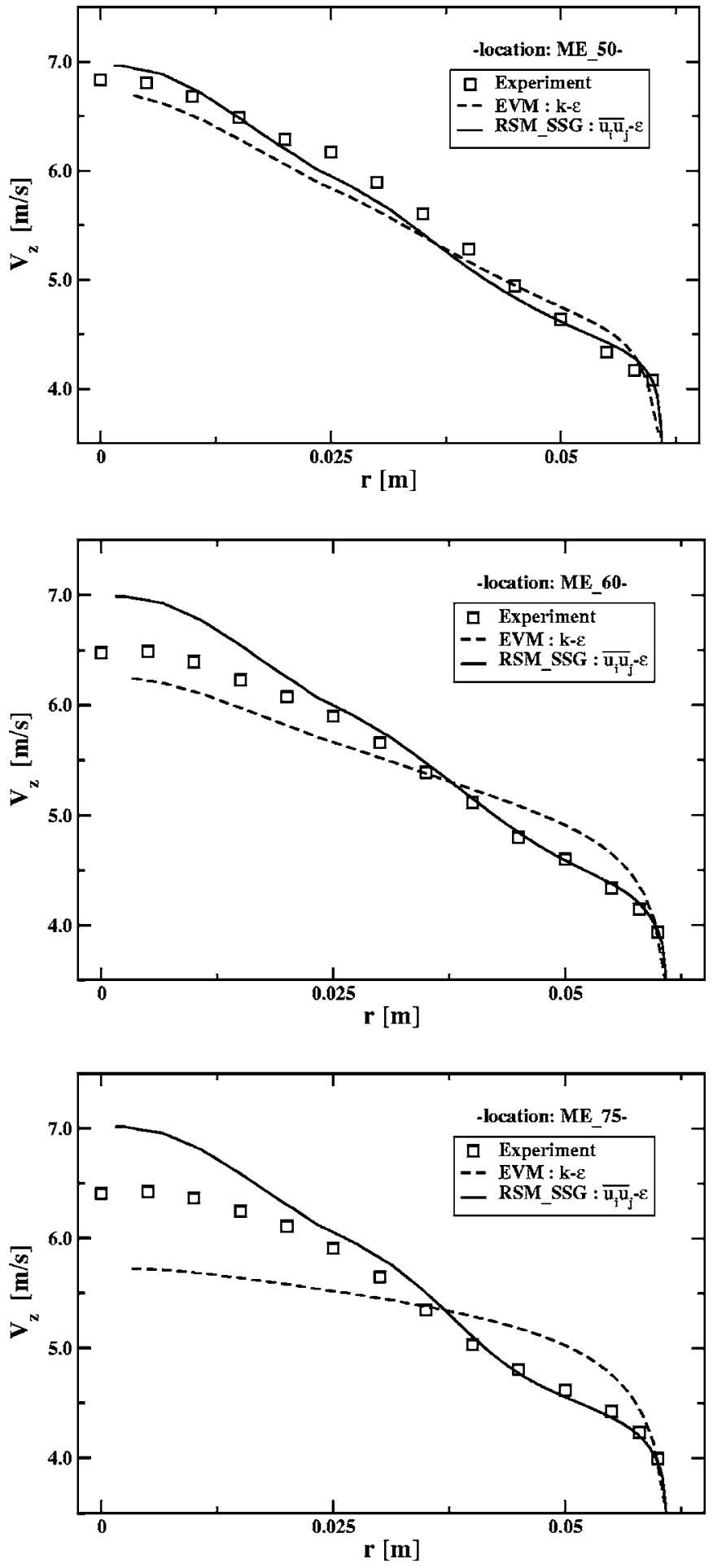

FIG. 4. Comparison of the axial velocity profiles in the inner cylinder of the 1:2 scale-down water model of the Riga dynamo setup at the ME50, ME60, and ME75 locations.

agreement with experimental profiles. The results of this model show peak values that are slightly overpredicted (indicating slower decay rate of the swirl intensity) but the region close to the inner wall is very well reproduced at all locations. The eddy-viscosity model performs quite reasonable too-although showing stronger decay of the swirl intensity as well as the overprediction of the axial velocity in the inner-wall regions-particularly for the last location 

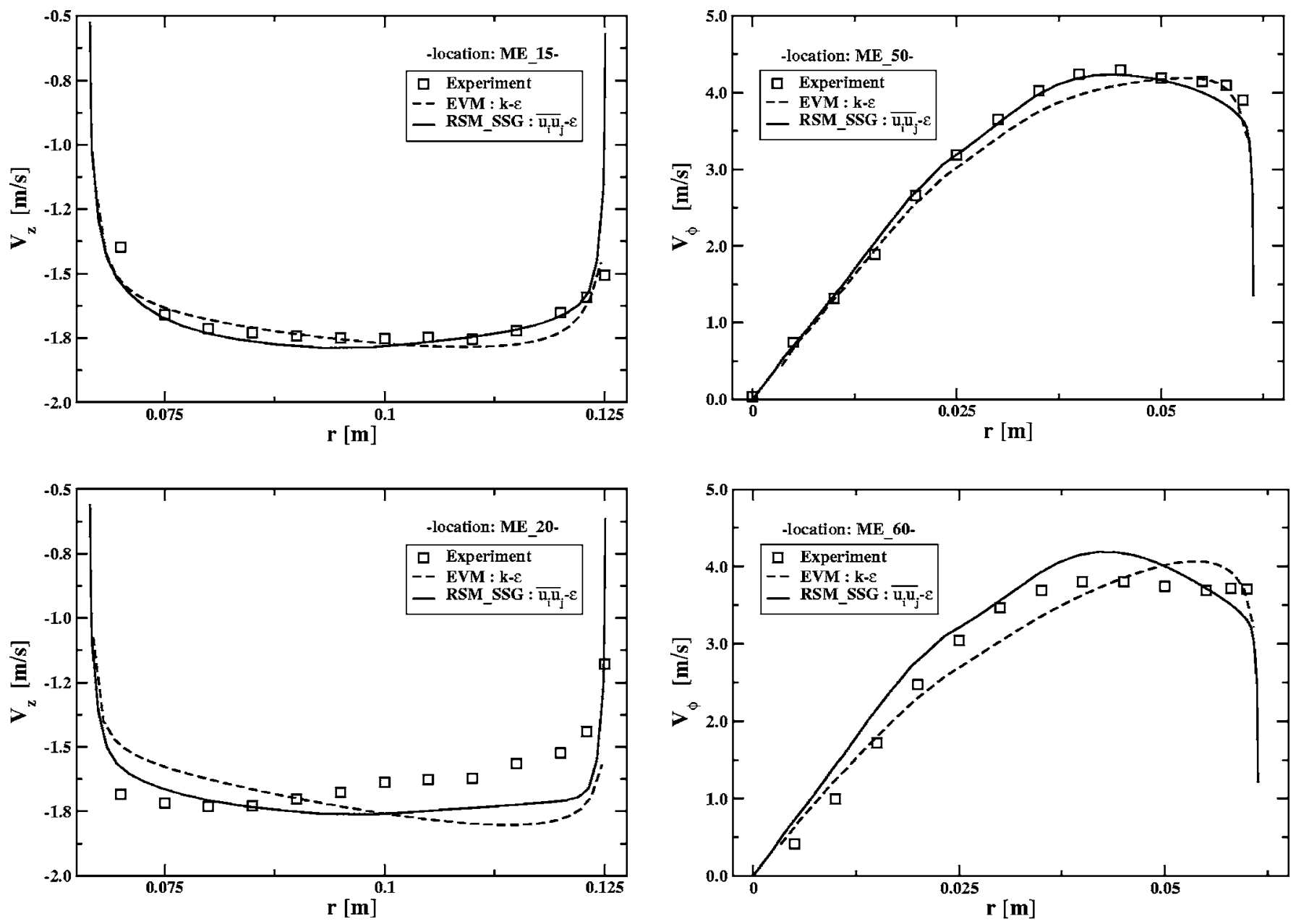

FIG. 5. Comparison of the axial velocity profiles in the outside passage of the 1:2 scale-down water model of the Riga dynamo setup at the ME15 and ME20 locations.

(ME75). For locations in the outer passage, both models show good agreement with experiments (Fig. 5). The azimuthal velocity distributions in the inner cylinder have the characteristic shape with zero values along the central line $(r=0)$ and peak values in the wall proximity (Fig. 6). It can be seen that both models produced good agreement with experiments. The peak values are, in contrast to the axial velocity, very well predicted at all locations. The only difference is in the locations of the peaks, which are again predicted by the second-moment closure in better agreement with experiments. A small underprediction in comparison with experiments is observed in the near-wall region where the eddy-viscosity model surprisingly shows slightly better agreements for the last two locations (ME60 and ME75).

It is important to mention that the azimuthal velocity component proved to be very sensitive to the applied numerical resolution and used numerical schemes. Application of the lower-level accuracy schemes (like upwind deferred schemes) produced significantly reduced values of the peak azimuthal velocity (approximately 50\%). These discrepancies are eliminated by using the TVD differencing scheme with the UMIST limiter for all variables. ${ }^{50}$

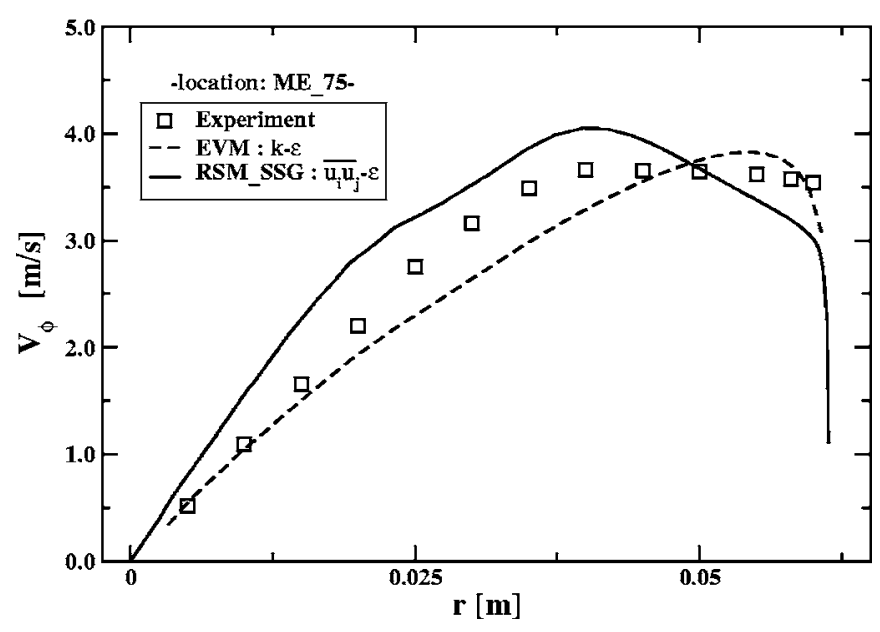

FIG. 6. Comparison of the azimuthal velocity profiles in the inside cylinder of the 1:2 scale-down water model of the Riga dynamo setup at the ME50, ME60, and ME75 locations.

It can be concluded that for the 1:2 scale-down water model good agreements are obtained between numerical simulations and measurements of the velocity field in the bulk of the inner and outer passage. The second-moment closure predictions were shown to be generally superior when compared with the simpler $k-\varepsilon$ model, and presumably, the superiority will be even more visible in the bend area and when comparing variables. But we note that the eddy- 
viscosity results are not much inferior especially when considered in light of satisfactory predictions of the azimuthal velocity component (Fig. 6), which has the most profound effect on the self-excitation phenomenon. The success of the $k-\varepsilon$ model can be explained by its tendency to produce a solid-body-rotation type of azimuthal velocity in swirling flow irrespective of the swirl origin, which has been regarded as an illustration of its inaptitude for swirling flows in general. However, in the present situation the imposed swirl is made on purpose to be close to solid-body rotation, thus suited for eddy-viscosity models. Then the problem remains, of course, if the back reaction of the magnetic field could alter significantly the solid-body-like profile to another profile for which the $k-\varepsilon$ model might not be appropriate. This scenario is rather unlikely since quite a number of experimental data (growth rates, frequencies, axial field dependence) support the general picture that the basic saturation effect comes from the downward braking of the azimuthal velocity component rather than from a radial redistribution of the flow. If the main saturation effect were due to a significant change of the radial profile of the azimuthal component, this would not result in the observed change of the axial dependence of the magnetic field as it was documented in Refs. 7 and 11-13. Moreover, since the main task of these numerical simulations is to provide the velocity perturbation in the saturated regime for the magnetic-field solver, the improvements achieved with the second-moment closure do not seem to be of crucial importance. Recalling also that the inclusion of the electromagnetic effects in second-moment closures requires substantial modeling and computational effort, it was decided that the simple two-equation $k-\varepsilon$ eddyviscosity model can serve the purposes of mimicking the saturation regime by using two separate numerical codes.

\section{Simulations of the full-scale experimental setup with sodium}

Next, we move to the simulations of the real-scale experimental setup with sodium as the working fluid. In addition to the active-fluid areas, i.e., the inner and outer passages, the surrounding annular layer of sodium initially at rest has also been taken into account. Both the vertical and horizontal cross planes with underlying numerical mesh are shown in Fig. 7. It can be seen that regions with significant velocity gradients (the near-wall regions and those with strong curvature) are additionally refined by mesh clustering. As in the previous case of the scaled-down water model, the entire closed loop is simulated. The eddy-viscosity $k-\varepsilon$ model is applied for the turbulence closure. The secondorder TVD numerical scheme (with UMIST limiter) is applied for all variables.

The first simulation run is performed in order to get the velocity field in the kinematic regime. The propeller working conditions of $2000 \mathrm{rpm}$ are specified. Fully developed timeaveraged (steady) results are shown in Fig. 8. The axial velocity component exhibits the typical pattern with positive values in the inner cylinder and with negative values in the outer passage. The azimuthal velocity component shows a gradual decrease in the inner cylinder from the imposed strong swirl at the propeller exit, and almost disappears in
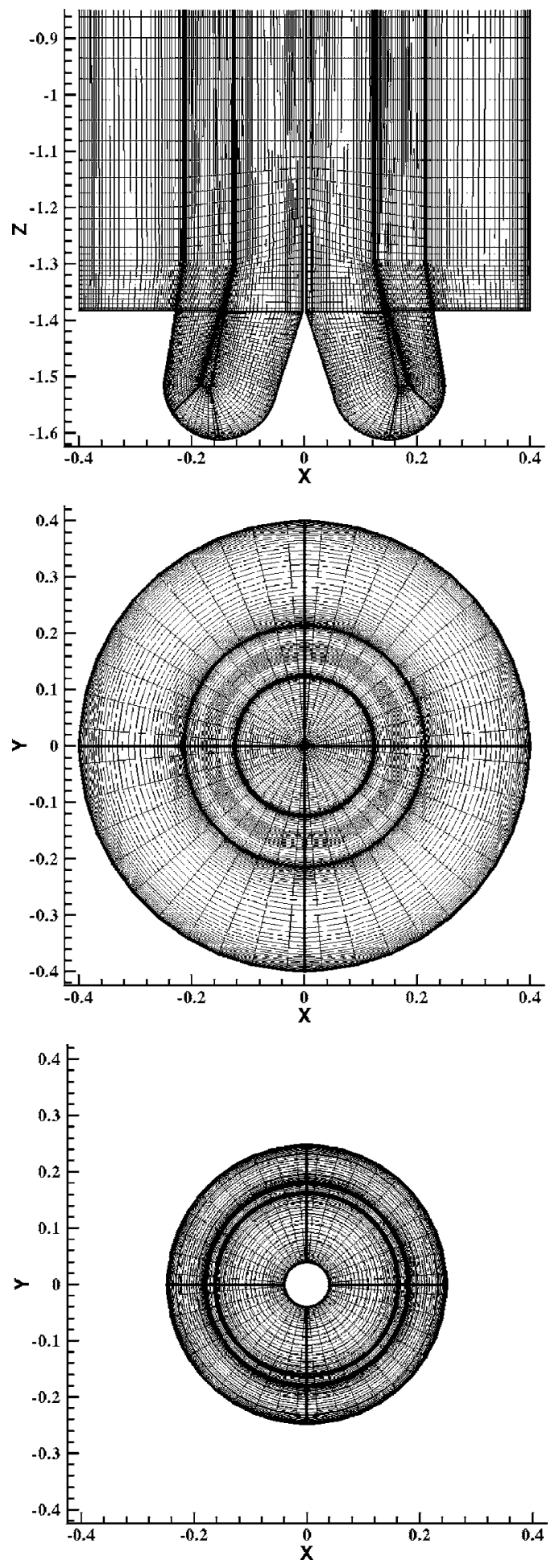

FIG. 7. Numerical mesh used for the discretization of the flow domains in the real-size Riga dynamo setup: central vertical cross section (top), horizontal cross section at $\mathrm{y}=-1 \mathrm{~m}$ (middle) and horizontal cross section at $\mathrm{y}=-1.5 \mathrm{~m}$ (below).

the outer passage as a consequence of the imposed mimicking of static guide vanes in the lower bend. From the turbulent kinetic-energy contours it is observed that the strong turbulence is associated with strong streamline curvature. The distribution of the eddy viscosity indicates that in the inner cylinder the flow is highly turbulent while the intensity 

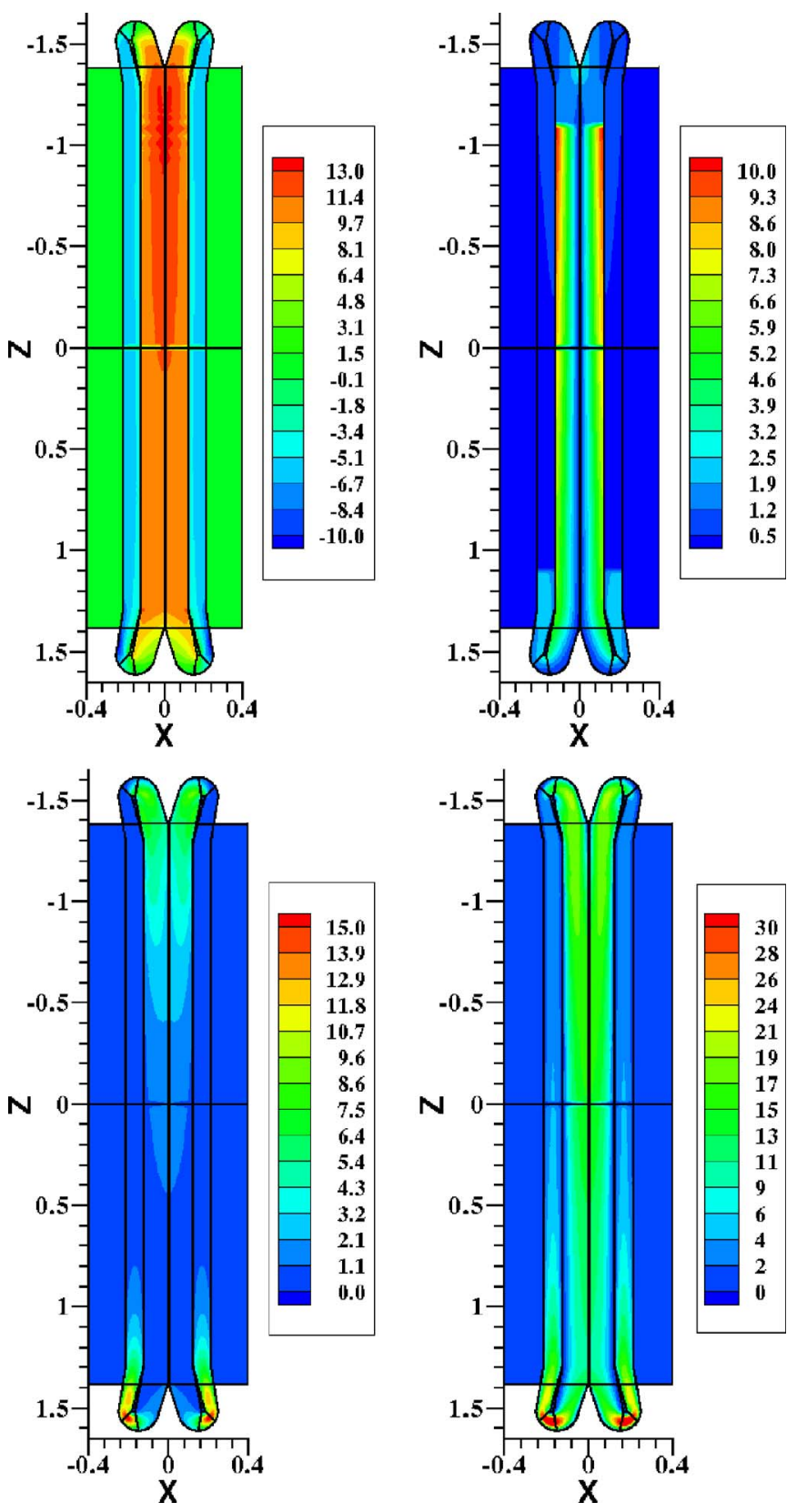

FIG. 8. (Color online) Axial and azimuthal velocity components [in $(\mathrm{m} / \mathrm{s})]$ above, turbulent kinetic energy [in $\left.\left(\mathrm{m}^{2} / \mathrm{s}^{2}\right)\right]$ and eddy viscosity $\left[\right.$ in $\left.\left(\mathrm{m}^{2} / \mathrm{s}\right)\right]$ below contour distributions in the central vertical plane of the real-size Riga dynamo setup: $\operatorname{Re}=3.5 \times 10^{6}$ (propeller working conditions of $2000 \mathrm{rpm}$ ) all before a magnetic field is generated.

of turbulence is gradually decreasing in the outer passage. Despite the high Reynolds number simulated $(\operatorname{Re} \approx 3.5$ $\times 10^{6}$ ) and the very complex geometry, simulations proved to be numerically very stable and high convergence criteria $\left(\epsilon_{\Phi}<10^{-6}\right)$ is achieved without difficulties for all variables. For this particular simulation, we used multiblock decomposition with 32 CPUs and almost ideal load balancing between processors is achieved at the SGI Origin 3800 system with 1024 CPUs.

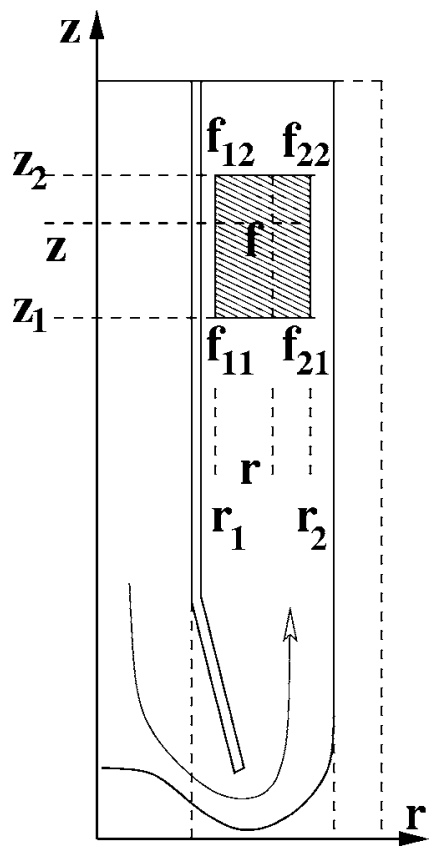

FIG. 9. Schematic of the interpolation between the $2 \mathrm{D}$ rectangular polarcylindrical magnetic-field solver (domain defined by dashed lines and a control volume defined by the dashed area) and the 3D nonorthogonal fluidflow solver (defined by solid lines).

\section{THE COUPLING OF THE CODES}

\section{A. Exchange of calculated fields between solvers}

Now, after completing the first set of the RANS-based fluid-flow and turbulence simulations, the coupling of the fluid-flow and magnetic-field solvers will be discussed. The magnetic-field code is used to get values of the electromagnetic parameters, including the magnetic field and Lorentz force components. Here it is important to note that exchange of the information between solvers is not a trivial task. The difference in definitions of local coordinate systems (the rectangular 2D polar cylindrical for the magnetic-field solver and the 3D nonorthogonal with Cartesian components) requires that additional effort must be put into this two-way indirect coupling of the solvers. In addition, the collocated locations of variables requested by the fluid-flow solver do not coincide with the discretized locations for variables used by the magnetic-field solver. The principles of the interpolation are demonstrated in Fig. 9, where the value of the arbitrary variable $(f)$ located in the center of the 3D nonorthogonal control volume are estimated as

$$
\begin{aligned}
f= & \frac{z_{2}-z}{z_{2}-z_{1}}\left(\frac{r_{2}-r}{r_{2}-r_{1}} f_{11}+\frac{r-r_{1}}{r_{2}-r_{1}} f_{21}\right) \\
& +\frac{z-z_{1}}{z_{2}-z_{1}}\left(\frac{r_{2}-r}{r_{2}-r_{1}} f_{12}+\frac{r-r_{1}}{r_{2}-r_{1}} f_{22}\right) .
\end{aligned}
$$

This approach is applied for all electromagnetic variables, i.e., $B_{i}, F_{i}^{L}$. Since a very fine numerical mesh was used for both numerical solvers $(225 \times 44$ rectangular nodes for the magnetic-field solver and the previously defined numerical mesh for the fluid-flow solver, Fig. 7), it was possible to obtain smooth interpolated fields without significant loses in 

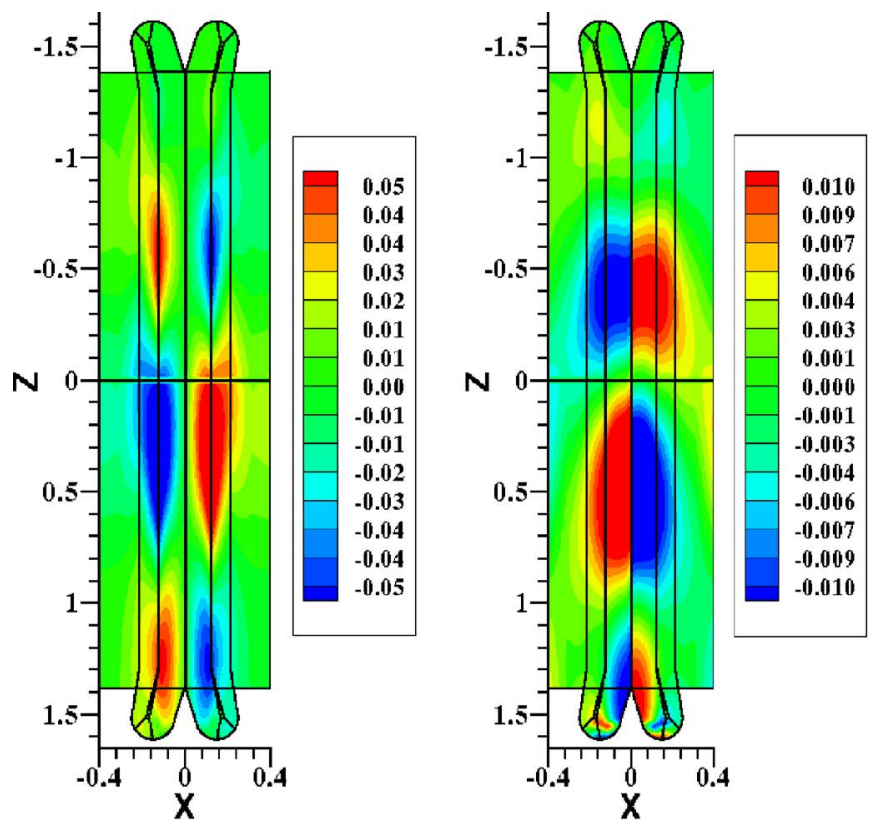

(A16)]. It should be stressed, however, that in the present realization of the coupling only the azimuthal average (the $m=0$ mode) over the Lorentz force has been taken into account while the $m=2$ mode has been neglected. This $m=2$ mode, which results from the quadratic appearance of the magnetic-field $m=1$ mode in the Lorentz force, would be needed to explain nonaxisymmetric velocity perturbations. This is also left for future simulations.

\section{B. An improved saturation model}

In this section we show how the coupling of both codes can be employed for a refinement of the former saturation model that was based on a simple one-dimensional backreaction model. ${ }^{11,14}$ Let us start by saying what a saturation model is. A dynamo is in a saturated state when the Lorentz forces, according to the self-excited magnetic field, modify the flow in such a way that the resulting growth rate of the magnetic field is just zero. Translated to the concrete Riga dynamo experiment, this means the following: for a certain supercritical rotation rate of the propeller, we measure the Joule excess power and infer from that the magnetic-field amplitude which cannot be obtained from purely kinematic simulation. This magnetic eigenfield is then plugged into the RANS solver for the velocity field, and the resulting flow modification is evaluated if it really leads to a zero growth rate. In addition, the eigenfield structure resulting from the modified velocity field can also be compared with the measured one.

Actually, this simple back-and-forth method represents only the first step of a perturbation method. The next step would be to compute again the magnetic field and Lorentz forces from the modified velocity, and so on.

Even such a simple-seeming back-reaction model is not without intricacies, in particular concerning a reliable determination of the Joule excess power. These problems were discussed in Ref. 14, where we have finally obtained a linear model of the form $P_{\text {Joule }}=(\Omega-1840 \mathrm{rpm}) /(1840 \mathrm{rpm})$ $\times 48.4 \mathrm{~kW}$, which fits reasonably to the available excess power data. It is worthwhile to note the very flat increase of the excess power with the rotation rate, particularly when compared with the steep power (i.e., pressure) increase in the Karlsruhe experiment (Ref. 15, inset of Fig. 4). The reason for this behavior is the freedom of the azimuthal velocity component to be braked by the Lorentz force. Now, having calibrated the magnetic field at a given propeller rotation rate, we use the RANS model to compute the flow modification due to the Lorentz force corresponding to this field.

In order to demonstrate the back-reaction effects of the imposed Lorentz force on the fluid flow, characteristic velocity profiles are shown in Figs. 11-13. For all figures, the Joule dissipation was chosen as $10 \mathrm{~kW}$. The profiles of the axial velocity components along the inner cylinder undergo significant changes when the effects of the imposed Lorentz force are taken into account. Note that the locations at which the profiles are extracted are defined with respect to the distance from the vertical center of the experimental setup so that all locations above the center have a positive sign $(+950,+786,+321 \mathrm{~mm})$ and those below this plane have a 

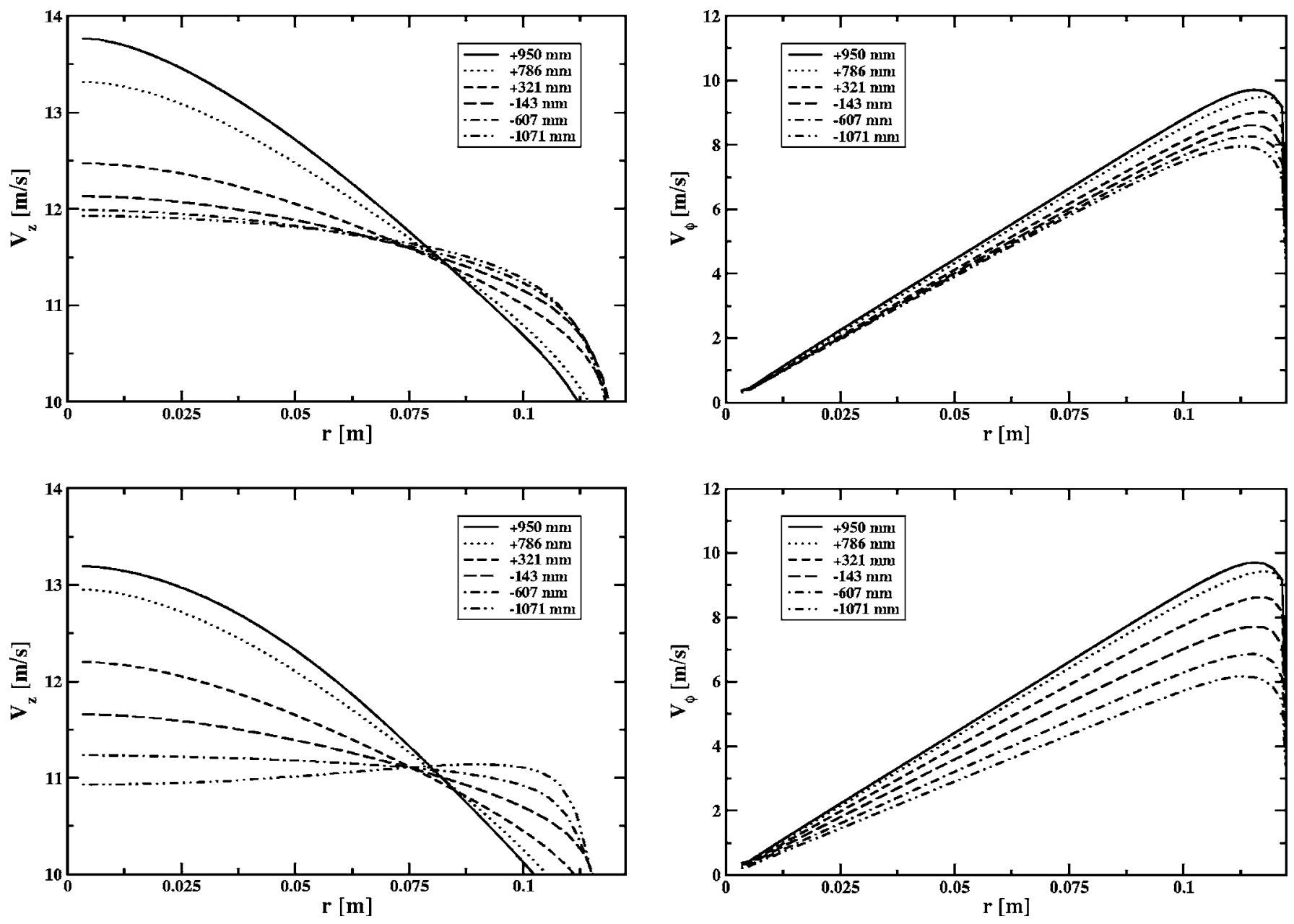

FIG. 11. Profiles of the axial velocity component along the inner cylinder without (above) and with (below) Lorentz force effects: propeller working conditions of $2000 \mathrm{rpm}$ and Joule dissipation of $10 \mathrm{~kW}$.

negative sign $(-143,-607,-1071 \mathrm{~mm})$. In addition to a stronger suppression in the central region close to the axis (indicating a faster rate of decay of the swirling intensity), the Lorentz force produces the characteristic " $M$ "-shaped profiles with distinct peaks in the wall proximity for the last two positions $(-607,-1071 \mathrm{~mm})$; Fig. 11. The azimuthal velocity profiles along the inner cylinder are more monotonic with characteristic peak values at the wall side (Fig. 12). Again, due to the active Lorentz force, the maximum values are significantly reduced.

The profiles of the axial velocity in the outer passage with and without the Lorentz force are significantly different (Fig. 13). These changes are caused both by different approaching profiles, which are particularly sensitive to the strong $180^{\circ}$ bend curvature, and the direct Lorentz force effects in the outer passage.

In the following step we have taken the computed velocity differences resulting from either the one-dimensional or the RANS model. Actually, this has been done for four different propeller rotation rates: 2000, 2200, 2400, and $2600 \mathrm{rpm}$. The amplitudes of the velocity changes were fixed in such a way that they corresponded to a Joule dissipation of $4.3 \mathrm{~kW}$ for $2000 \mathrm{rpm}$, of $9.5 \mathrm{~kW}$ for $2200 \mathrm{rpm}$, of $14.7 \mathrm{~kW}$

FIG. 12. Profiles of the azimuthal velocity component along the inner cylinder without (above) and with (below) Lorentz force effects (identical working conditions as in previous figure).

for $2400 \mathrm{rpm}$, and of $20 \mathrm{~kW}$ for $2600 \mathrm{rpm}$. The velocity perturbations for a Joule dissipation of $9.5 \mathrm{~kW}$ are shown in Fig. 14 for the central cylinder, and in Fig. 15 for the backflow annular passage. In the inner channel, it is remarkable that the profiles of $v_{\varphi}$ in the one-dimensional and in the RANS models are very close to each other. In the outer channel this difference is a bit larger, but not dramatic. The RANS model makes also checkable predictions on the deformation of the axial velocity, which was not possible by the simple onedimensional model that did not include any change of $v_{z}$.

For each of the propeller rotation rates, 2000, 2200, 2400 , and $2600 \mathrm{rpm}$, we have computed the growth rates and the frequencies (see Fig. 16) with the two back-reaction models (1D and RANS). What can we learn from Fig. 16? When looking at the growth rate curve we see that the main effect (i.e., going down from the kinematic curve to the saturated curve) is already included in the one-dimensional model. The RANS model brings the curve even closer to the zero line, improving therefore the saturation model. In contrast to the growth rate, the frequency does not decrease. It even increases slightly in comparison to what would be expected from extrapolating the kinematic regime. Although not documented here, the saturation model is also in good 

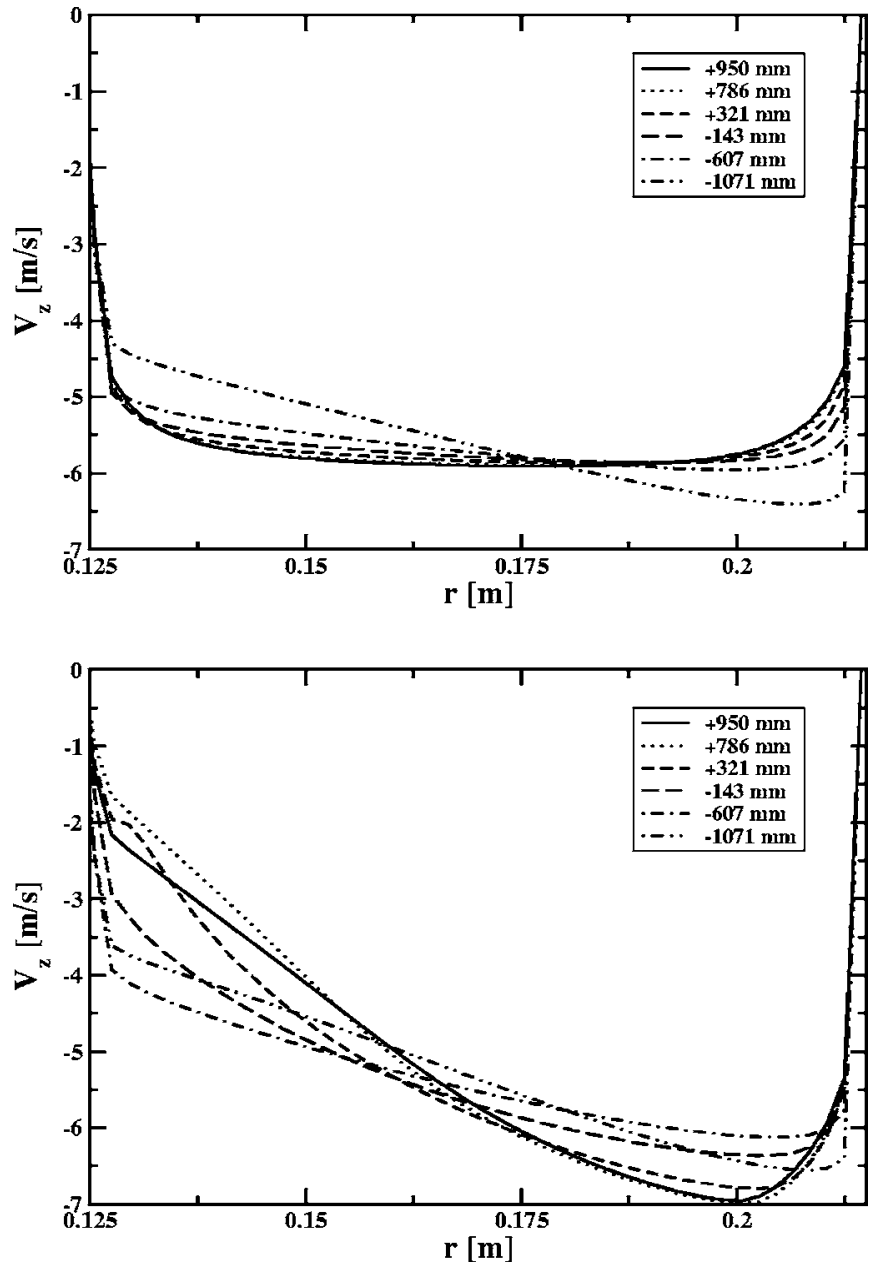

FIG. 13. Profiles of the axial velocity component in the outer annular passage without (above) and with (below) Lorentz force effects.

agreement with the observed shift of the magnetic-field structure towards the propeller region. ${ }^{11}$ This change in the field structure mirrors the deteriorated excitation ability in the lower part of the dynamo due to the braked azimuthal velocity. The effect of the RANS model on the frequency is not significantly different from that of the one-dimensional model.

\section{CONCLUSIONS AND OUTLOOK}

We studied numerically the mechanism of the magneticfield generation in the Riga dynamo experiment, aimed at gaining further insight into the velocity and magnetic field and their interaction in the saturation regime. This regime, characterized by the zero growth rate of the generated magnetic field, was simulated by using a coupled RANS solver for the fluid flow (Navier-Stokes) and a finite-difference solver for the magnetic field.

First, we have obtained the velocity and turbulence fields in the kinematic regime, i.e., without magnetic field. The fields and Lorentz forces resulting from the magnetic-field solver were exported to the fluid solver where all additional model terms associated with electromagnetic effects are activated. Then, the velocity perturbations obtained under the influence of the Lorentz force were exported back into the
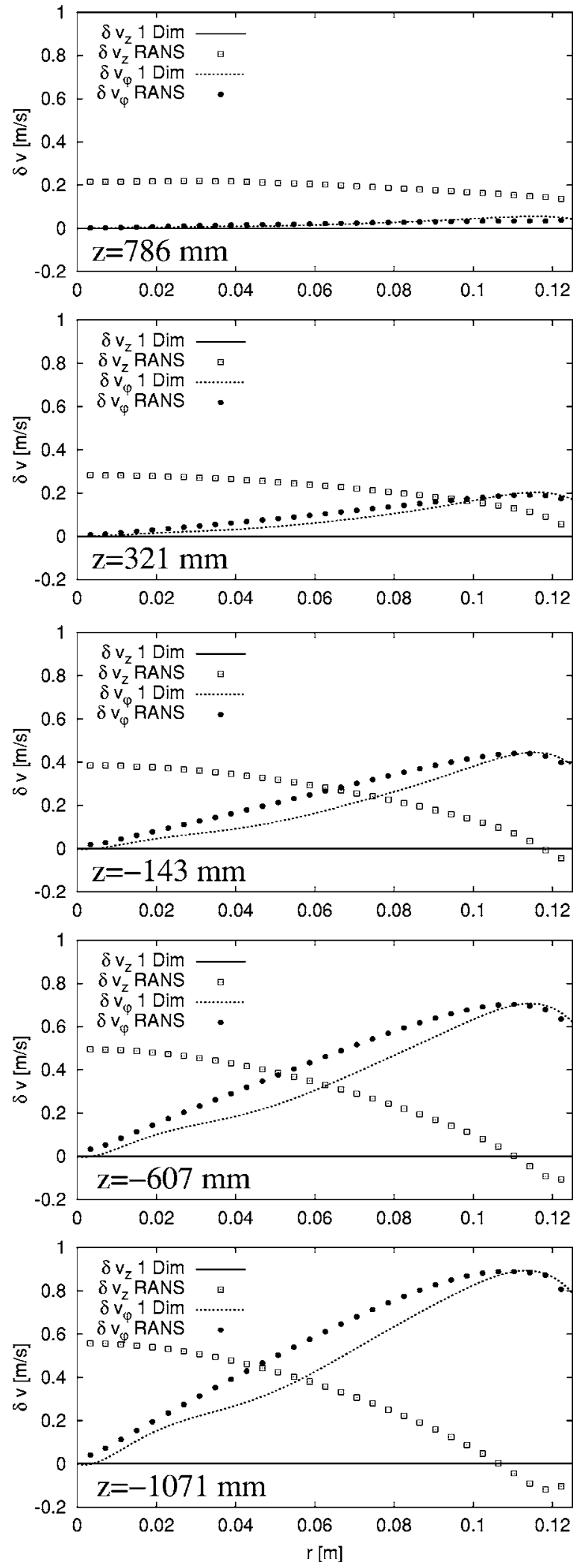

FIG. 14. Differences of velocity profiles with and without Lorentz forces: inner cylinder.

magnetic-field solver in order to capture the differences between the kinematic and the saturated regimes. The exchange of fields between solvers was done by multidimensional interpolation/extrapolation technique, which provided accu- 

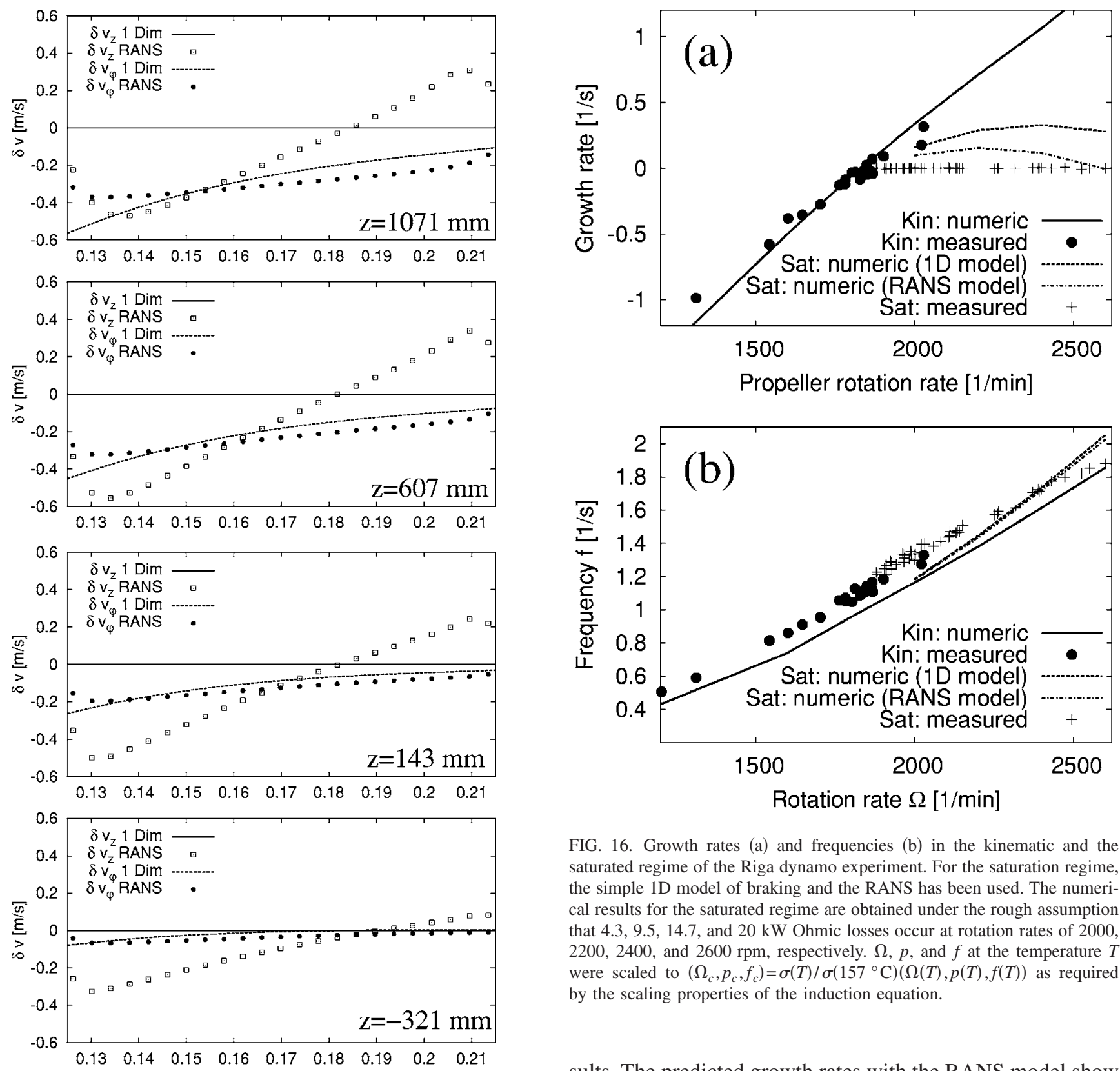

FIG. 16. Growth rates (a) and frequencies (b) in the kinematic and the saturated regime of the Riga dynamo experiment. For the saturation regime, the simple 1D model of braking and the RANS has been used. The numerical results for the saturated regime are obtained under the rough assumption that $4.3,9.5,14.7$, and $20 \mathrm{~kW}$ Ohmic losses occur at rotation rates of 2000, 2200, 2400, and $2600 \mathrm{rpm}$, respectively. $\Omega, p$, and $f$ at the temperature $T$ were scaled to $\left(\Omega_{c}, p_{c}, f_{c}\right)=\sigma(T) / \sigma\left(157^{\circ} \mathrm{C}\right)(\Omega(T), p(T), f(T))$ as required by the scaling properties of the induction equation.

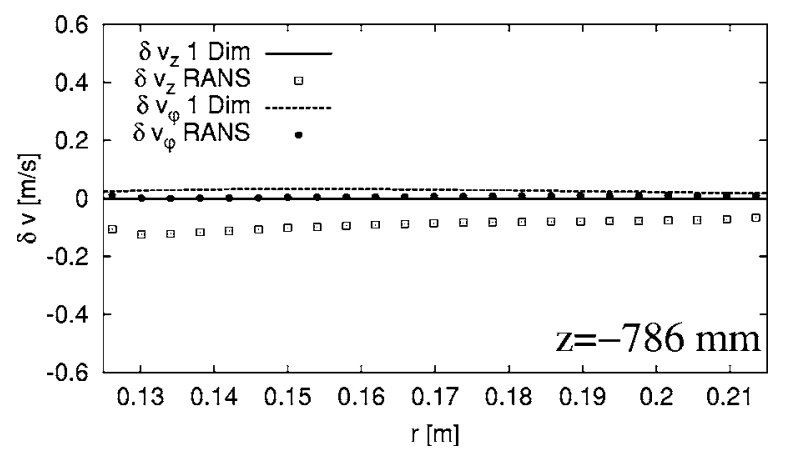

FIG. 15. Differences of velocity profiles with and without Lorentz forces: outer cylinder.

rate projections with smooth fields. These computations were performed for different propeller rotation rates, and the growth rates and frequencies of the self-excited magneticfield components are compared with the experimental re-

sults. The predicted growth rates with the RANS model show an improvement over the simple one-dimensional saturation models. In contrast, the predicted frequencies are just marginally different from these predicted by the one-dimensional model.

Despite significant simplification and approximation, especially in treating the magnetic field and sequential interuse of two solvers, the simulations have reproduced the major parameters - the growth rate and frequencies of the Riga experiment in close accord with measurements. Useful new information about the fluid flow and magnetic fields and their interaction have also been obtained. A main novelty of the RANS model compared with the former one-dimensional model is that a radial restructuring of the axial velocity profile has been predicted. It would certainly be interesting to detect this effect by flow measurements. Of course, more comprehensive information, especially about the selfexcitation dynamics, could be expected only from the simultaneous time solutions for both fields accounting for direct two-way coupling, which is currently in progress. 
TABLE I. Specification of coefficients in $\overline{u_{i} u_{j}}$ and $\varepsilon$ equations in the presence of the imposed electromagnetic fields for Speziale et al. (Ref. 48) and Kenjereš et al. (Ref. 45) second-moment closure with MHD terms.

\begin{tabular}{lllllllllllll}
\hline \hline$C_{1}$ & $C_{1}^{*}$ & $C_{2}$ & $C_{3}$ & $C_{3}^{*}$ & $C_{3}^{M}$ & $C_{4}$ & $C_{5}$ & $C_{S}$ & $C_{\varepsilon}$ & $C_{\varepsilon 1}$ & $C_{\varepsilon 2}$ & $C_{\lambda}$ \\
3.4 & 1.8 & 4.2 & 0.8 & 1.3 & 0.6 & 1.25 & 0.4 & 0.22 & 0.18 & 1.44 & 1.83 & 0.6 \\
\hline \hline
\end{tabular}

\section{ACKNOWLEDGMENTS}

Access to the supercomputing facilities at the SARA Computing and Networking Services in Amsterdam has been provided by the National Computing Facilities Foundation (NCF) and sponsored by the Nederlandse Organisatie voor Wetenschappelijk Onderzoek (NWO). We thank Michael Christen (TU Dresden) for providing velocity data from the water test facility. The results presented in this paper emerged from the research project "MAGDYN: Magnetic Field Dynamos-Laboratory Studies based on the Riga Dynamo Facility" sponsored by the European Commission under Contract No. HPRI-CT-2001-50027. The research of S.K. has been made possible through a fellowship of the Royal Netherlands Academy of Arts and Sciences (KNAW). This work was supported by Deutsche Forschungsgemeinschaft in the framework of SFB 609 and Grant No. GE 682/ 14-1.

\section{APPENDIX}

\section{Second-moment (Reynolds-stress) model for MHD}

This model includes the solving of all turbulent-stress components $\left(\overline{u_{i} u_{j}}\right)$ together with an equation for the dissipation rate of the turbulent kinetic energy $(\varepsilon)$,

$$
\begin{aligned}
\frac{\partial \overline{u_{i} u_{j}}}{\partial t}+U_{l} \frac{\partial \overline{u_{i} u_{j}}}{\partial x_{l}}= & \frac{\partial}{\partial x_{l}}\left[\left(\nu \delta_{l m}+C_{s} \frac{k}{\varepsilon} \overline{u_{l} u_{m}}\right) \frac{\partial \overline{u_{i} u_{j}}}{\partial x_{m}}\right]+P_{i j} \\
& +\Phi_{i j}^{S}+\Phi_{i j}^{R}+\Phi_{i j}^{M}-\varepsilon_{i j}+S_{i j}^{M}, \\
\frac{\partial \varepsilon}{\partial t}+U_{l} \frac{\partial \varepsilon}{\partial x_{l}}= & \frac{\partial}{\partial x_{l}}\left[\left(\nu \delta_{l m}+C_{\varepsilon} \frac{k}{\varepsilon} \overline{u_{l} u_{m}}\right) \frac{\partial \varepsilon}{\partial x_{m}}\right]+C_{\varepsilon 1} \frac{\varepsilon}{k} P \\
& -C_{\varepsilon 2} \frac{\varepsilon^{2}}{k}+S_{\varepsilon}^{M},
\end{aligned}
$$

where

$$
P_{i j}=-\overline{u_{i} u_{l}} \frac{\partial U_{j}}{\partial x_{l}}-\overline{u_{j} u_{l}} \frac{\partial U_{i}}{\partial x_{l}}, \quad P=-\overline{u_{i} u_{j}} \frac{\partial U_{i}}{\partial x_{j}}
$$

are the production of turbulent stresses and of turbulent kinetic energy, respectively, and $\delta_{l m}$ is the Kronecker delta. The additional "MHD" terms are included in their exact form,

$$
S_{i j}^{M}=S_{i j}^{M 1}+S_{i j}^{M 2},
$$

where

$$
S_{i j}^{M 1}=-\frac{\sigma}{\rho}\left(\epsilon_{i l m} B_{m} \overline{u_{j} \frac{\partial \varphi}{\partial x_{l}}}+\epsilon_{j l m} B_{m} \overline{u_{i} \frac{\partial \varphi}{\partial x_{l}}}\right)
$$

$$
S_{i j}^{M 2}=\frac{\sigma}{\rho}\left(B_{i} B_{l} \overline{u_{j} u_{l}}+B_{j} B_{l} \overline{u_{i} u_{l}}-2 B_{l}^{2} \overline{u_{i} u_{j}}\right) .
$$

The correlation between fluctuating velocity components and gradients of the electric potential is modeled as

$$
\overline{u_{i} \frac{\partial \varphi}{\partial x_{j}}}=C_{\lambda} \epsilon_{j l m} B_{m} \overline{u_{i} u_{l}},
$$

where $\epsilon_{j l m}$ is the permutation tensor. Finally, slow, rapid, and electromagnetic parts of the pressure-strain correlation are modeled as

$$
\begin{aligned}
\Phi_{i j}^{S}= & -\left(C_{1} \varepsilon+C_{1}^{*} P b_{i j}\right), \\
\Phi_{i j}^{R}= & C_{2} \varepsilon\left(b_{i j}^{2}-\frac{1}{3} b_{l l}^{2} \delta_{i j}\right)+S_{i j} k\left(C_{3}-C_{3}^{*}\left(b_{i j} b_{j i}\right)^{1 / 2}\right) \\
& +C_{4} k\left(S_{i l} b_{l j}+S_{l j} b_{i l}-\frac{2}{3} S_{l m} b_{m l} \delta_{i j}\right) \\
& +C_{5} k\left(\Omega_{i l} b_{l j}-\Omega_{l j} b_{i l}\right), \\
\Phi_{i j}^{M}= & -C_{3}^{M}\left(S_{i j}^{M}-\frac{2}{3} S^{M} \delta_{i j}\right),
\end{aligned}
$$

where

$$
\begin{aligned}
& S^{M}=\frac{1}{2} S_{i i}^{M}, \quad b_{i j}=\frac{\overline{u_{i} u_{j}}}{2 k}-\frac{1}{3} \delta_{i j}, \\
& S_{i j}=\frac{1}{2}\left(\frac{\partial U_{i}}{\partial x_{j}}+\frac{\partial U_{j}}{\partial x_{i}}\right), \quad \Omega_{i j}=\frac{1}{2}\left(\frac{\partial U_{i}}{\partial x_{j}}-\frac{\partial U_{j}}{\partial x_{i}}\right)
\end{aligned}
$$

are the magnetic turbulent kinetic-energy production, the anisotropy, the mean rate of strain, and the mean vorticity tensors, respectively. This final version of the model is essentially a combination of the Speziale et al. ${ }^{48}$ second-moment closure with MHD extensions proposed by Kenjereš et al. ${ }^{46}$ Finally, all model coefficients are given in Table I.

\section{The $k-\varepsilon$ model with MHD effects}

The simplified version of the above model can be obtained by contracting indices, i.e., $k=0.5 \overline{u_{i} u_{i}}$ and by introducing the turbulent viscosity $\left(\nu_{t}\right)$ as

TABLE II. Specification of coefficients in $k$ and $\varepsilon$ equations in the presence of the imposed electromagnetic fields for simplified eddy-viscosity model with MHD terms.

\begin{tabular}{lccccc}
\hline \hline$\sigma_{k}$ & $\sigma_{\varepsilon}$ & $c_{\mu}$ & $C_{\varepsilon 1}$ & $C_{\varepsilon 2}$ & $C_{1}^{M}$ \\
1. & 1.3 & 0.09 & 1.44 & 1.92 & 0.025 \\
\hline \hline
\end{tabular}




$$
\begin{aligned}
\overline{u_{i} u_{j}}=\frac{2}{3} k \delta_{i j}-\nu_{t}\left(\frac{\partial U_{i}}{\partial x_{j}}+\frac{\partial U_{j}}{\partial x_{i}}\right), \quad \nu_{t}=c_{\mu} k^{2} / \varepsilon \\
\frac{\partial k}{\partial t}+U_{j} \frac{\partial k}{\partial x_{j}}=\frac{\partial}{\partial x_{j}}\left[\left(\nu+\frac{\nu_{t}}{\sigma_{k}}\right) \frac{\partial k}{\partial x_{j}}\right]+P-\varepsilon+S^{M}, \\
\frac{\partial \varepsilon}{\partial t}+U_{j} \frac{\partial \varepsilon}{\partial x_{j}}=\frac{\partial}{\partial x_{j}}\left[\left(\nu+\frac{\nu_{t}}{\sigma_{\varepsilon}}\right) \frac{\partial \varepsilon}{\partial x_{j}}\right]+C_{\varepsilon 1} \frac{\varepsilon}{k} P-C_{\varepsilon 2} \frac{\varepsilon^{2}}{k} \\
+S_{\varepsilon}^{M},
\end{aligned}
$$

where the production of turbulent kinetic energy is modeled as

$$
P=\nu_{t}|S|^{2}, \quad|S|=\sqrt{2 S_{i j} S_{i j}},
$$

and additional MHD terms are included as

$$
S^{M}=-\frac{\sigma}{\rho} B_{0}^{2} k \exp \left(-C_{1}^{M} \frac{\sigma}{\rho} B_{0}^{2} \frac{k}{\varepsilon}\right), \quad S_{\varepsilon}^{M}=S^{M} \frac{\varepsilon}{k} .
$$

This final version of the model is a combination of the standard two-equation $k-\varepsilon$ eddy-viscosity model with MHD extensions proposed in Kenjereš and Hanjalić. ${ }^{42}$ The coefficients for this $k-\varepsilon$ MHD model are given in Table II.

${ }^{1}$ H. K. Moffatt, Field Generation in Electrically Conducting Fluids (Cambridge University Press, Cambridge, London, New York, Melbourne, 1978).

${ }^{2}$ F. Krause and K.-H. Rädler, Mean-field Magnetohydrodynamics and Dynamo Theory (Pergamon, Oxford, 1980).

${ }^{3}$ G. Rüdiger and R. Hollerbach, The Magnetic Universe: Geophysical and Astrophysical Dynamo Theory (Wiley, Weinheim, 2004).

${ }^{4}$ I. Wilkinson, Geophys. Surv. 7, 107 (1984).

${ }^{5}$ A. Gailitis, O. Lielausis, S. Dement'ev, E. Platacis, A. Cifersons, G. Gerbeth, Th. Gundrum, F. Stefani, M. Christen, H. Hänel, and G. Will, Phys. Rev. Lett. 84, 4365 (2000).

${ }^{6}$ R. Stieglitz and U. Müller, Phys. Fluids 13, 561 (2001).

${ }^{7}$ A. Gailitis, O. Lielausis, E. Platacis, S. Dement'ev, A. Cifersons, G. Gerbeth, Th. Gundrum, F. Stefani, M. Christen, and G. Will, Phys. Rev. Lett. 86, 3024 (2001).

${ }^{8}$ A. Gailitis, O. Lielausis, E. Platacis, G. Gerbeth, and F. Stefani, Magnetohydrodynamics $\mathbf{3 7}, 71$ (2001).

${ }^{9}$ A. Gailitis, O. Lielausis, E. Platacis, G. Gerbeth, and F. Stefani, Dynamo and Dynamics, a Mathemetical Challenge, edited by D. Armbruster and I. Oprea (Kluwer, Dordrecht, 2001), pp. 9-16.

${ }^{10}$ A. Gailitis, O. Lielausis, E. Platacis, S. Dement'ev, A. Cifersons, G. Gerbeth, Th. Gundrum, F. Stefani, M. Christen, and G. Will, Magnetohydrodynamics 38, 5 (2002).

${ }^{11}$ A. Gailitis, O. Lielausis, E. Platacis, G. Gerbeth, and F. Stefani, Magnetohydrodynamics 38, 15 (2002).

${ }^{12}$ A. Gailitis, O. Lielausis, E. Platacis, G. Gerbeth, and F. Stefani, Rev. Mod. Phys. 74, 973 (2002).

${ }^{13}$ A. Gailitis, O. Lielausis, E. Platacis, G. Gerbeth, and F. Stefani, Surv. Geophys. 74, 973 (2002).
${ }^{14}$ A. Gailitis, O. Lielausis, E. Platacis, G. Gerbeth, and F. Stefani, Phys. Plasmas 11, 2838 (2004).

${ }^{15}$ R. Stieglitz and U. Müller, Magnetohydrodynamics 38, 27 (2002).

${ }^{16}$ U. Müller and R. Stieglitz, Nonlinear Processes Geophys. 9, 165 (2002).

${ }^{17}$ U. Müller, R. Stieglitz, and S. Horanyi, J. Fluid Mech. 498, 31 (2004).

${ }^{18}$ U. Müller, R. Stieglitz, and S. Horanyi, J. Fluid Mech. 552, 419 (2006).

${ }^{19}$ N. L. Peffley, A. B. Cawthorne, and D. P. Lathrop, Phys. Rev. E 61, 5287 (2000).

${ }^{20}$ F. Pétrélis, M. Bourgoin, L. Marié, J. Burguete, A. Chiffaudel, F. Daviaud, S. Fauve, P. Odier, and J. F. Pinton, Phys. Rev. Lett. 90, 174501 (2003).

${ }^{21}$ E. J. Spence, M. D. Nornberg, C. M. Jacobson, R. D. Kendrick, and C. B. Forest, Phys. Rev. Lett. 96, 055002 (2006).

${ }^{22}$ P. Frick, V. Noskov, S. Denisov, S. Khripchenko, D. Sokoloff, R. Stepanov, and A. Sukhanowsky, Magnetohydrodynamics 38, 143 (2002).

${ }^{23}$ H.-C. Nataf, T. Alboussiére, D. Brito, P. Cardin, N. Gagniére, D. Jault, J.-P. Masson, and D. Schmitt, Geophys. Astrophys. Fluid Dyn. (unpublished); http://arxiv.org/abs/physics/0512217.

${ }^{24}$ G. A. Glatzmaier, Annu. Rev. Earth Planet. Sci. 30, 237 (2002).

${ }^{25}$ B. A. Buffett, Geophys. J. Int. 153, 753 (2003).

${ }^{26}$ M. Matsushima, Phys. Earth Planet. Inter. 153, 74 (2005).

${ }^{27}$ F. Hamba, Phys. Plasmas 11, 5316 (2004).

${ }^{28}$ F. Stefani, G. Gerbeth, and A. Gailitis, in Transfer Phenomena in Magnetohydrodynamic and Electroconducting Flows, edited by A. Alemany, $\mathrm{Ph}$. Marty, and J. P. Thibault (Kluwer, Dordrecht, 1999), pp. 31-44.

${ }^{29}$ Y. Ponty, H. Politano, and J.-F. Pinton, Phys. Rev. Lett. 92, 144503 (2004).

${ }^{30}$ J.-L. Guermond, J. Léorat, and C. Nore, Eur. J. Mech. B/Fluids 22, 555 (2003).

${ }^{31}$ F. Stefani, G. Gerbeth, and K.-H. Rädler, Astron. Nachr. 321, 65 (2000).

${ }^{32}$ M. Xu, F. Stefani, and G. Gerbeth, J. Comput. Phys. 196, 102 (2004).

${ }^{33}$ M. Xu, F. Stefani, and G. Gerbeth, Phys. Rev. E 70, 056305 (2004).

${ }^{34}$ A. B. Iskakov, S. Descombes, and E. Dormy, J. Comput. Phys. 197, 540 (2004).

${ }^{35}$ A. Brandenburg, A. Nordlund, R. F. Stein, and U. Torkelsson, Astrophys. J. 446, 741 (1995).

${ }^{36}$ M. Christen, H. Hänel, and G. Will, in Beiträge zu Fluidenergiemaschinen 4, edited by W. H. Faragallah and G. Grabow (Faragallah-Verlag und Bildarchiv, Sulzbach/Ts., 1998), pp. 111-119.

${ }^{37}$ M. Christen (private communication).

${ }^{38}$ Yu. B. Ponomarenko, J. Appl. Mech. Tech. Phys. 14, 775 (1973).

${ }^{39}$ A. Gailitis and Ya. Freibergs, Magnetohydrodynamics (N.Y.) 12, 127 (1976).

${ }^{40}$ A. Gailitis and Ya. Freibergs, Magnetohydrodynamics (N.Y.) 16, 116 (1980).

${ }^{41}$ A. Gailitis, Magnetohydrodynamics (N.Y.) 32, 58 (1996).

${ }^{42}$ S. Kenjereš and K. Hanjalić, Int. J. Heat Fluid Flow 21, 329 (2000).

${ }^{43}$ K. Hanjalić and S. Kenjereš, J. Turbul. 1, 1 (2000).

${ }^{44}$ K. Hanjalić and S. Kenjereš, Flow, Turbul. Combust. 66, 427 (2001).

${ }^{45}$ S. Kenjereš and K. Hanjalić, Int. J. Heat Fluid Flow 25, 559 (2004).

${ }^{46}$ S. Kenjereš, K. Hanjalić, and D. Bal, Phys. Fluids 16, 1229 (2004).

${ }^{47}$ K. Hanjalić and S. Kenjereš, Trans. ASME, J. Appl. Mech. 73, 430 (2006).

${ }^{48}$ C. G. Speziale, S. Sarkar, and T. B. Gatski, J. Fluid Mech. 227, 245 (1991).

${ }^{49}$ M. M. Gibson and B. E. Launder, J. Fluid Mech. 86, 491 (1978).

${ }^{50}$ F. S. Lien and M. A. Leschziner, Int. J. Numer. Methods Fluids 19, 527 (1994). 